\title{
AN HYPERVALUATION OF A RING ONTO A TOTALLY ORDERED NON-CANCELLATIVE SEMIGROUP WITHOUT ZERO DIVISORS
}

\author{
JOHN PAPADOPOULOS \\ 41-43 Ioulianou str., Athens 10434 GREECE \\ (Received April 17, 1985)
}

ABSTRACT. In this paper we answer to a question posed by Marc KRANSER: It it possible to have a totally ordered noncancellative semigroup without zero divisors, and a ring hypervaluated by this semigroup? We were able to give a positive answer and provide an example.

KEY WORDS AND PHRASES. Hypervaluation, Valuation, Totally ordered semigroup, Ring 1980 AMS SUBJECT CLASSIFICATION CODE: 16A34 or 16A45.

\section{PRELIMINARIES}

In what follows, all semigroups are supposed to have a unit element 1 and a zero (absorbent) element 0 , such that $a \cdot 0=0 . a=0$ for all elements $a$ in the semigroup. In any semigroup we can adjoint a zero element if it does not already have one, without changing its structure. We remark that in each semigroup 1 and 0 are unique.

DEFINITION 1. We say that a semigroup $S$ is ordered if it is supplied with an order < such that:

1. For $\mathrm{a}, \mathrm{b}, \mathrm{c}$ in $\mathrm{S}, \mathrm{a}<\mathrm{b} \Rightarrow \mathrm{ca} \leq \mathrm{cb}$ and $\mathrm{ac} \leq \mathrm{bc}$.

2. $0<1$ (hence $0=0 c \leq 1 c=c$ for all $c$ in $\mathrm{S}$ )

If the order is total $\mathrm{S}$ is called totally ordered.

DEFINITION 2. An hypervaluation on a ring $R$ is a function from $R$ onto a totally ordered semigroup $\mathrm{S}$, satisfying the following conditions: For all $\mathrm{a}, \mathrm{b}$ in $\mathrm{R}$.

$$
\begin{aligned}
& \text { 1. }|a|=0 \Leftrightarrow a=0 \\
& \text { 2. }|a|=|-a| \\
& \text { 3. }|a b|=|a||b| \\
& \text { 4. }|a+b| \leq \operatorname{Max}\{|a|,|b|\}
\end{aligned}
$$

Notice that if the semigroup $S$ does not have any zero divisors then the ring $R$ does not have any either. For if $a, b \in R$ with $a \neq 0, b \neq 0$, and $a b=0$, then $0=|0|=|a b|=|a||b|$, while $|a| \neq 0$ and $|b| \neq 0$. But this is impossible since $S$ is assumed with no zero divisors. Also we easily see that a cancellative semigroup has no zero divisors, however the converse is not true in general as we shall see in what follows. 
2. CONSTRUCTION OF A NON-CANCELLATIVE, TOTALLY ORDERED SEMIGROUP WITHOUT ZERO DIVISORS

We begin with an arbitrary given totally ordered semigroup $\left(S_{1}, \cdot,>\right)=\left\{0_{1}, a, b, \ldots\right\}$ where $0_{1}$ its absorbent (zero) element. Consider now the set $\mathrm{s}_{2}=\mathrm{S}_{1} \mathrm{U}\left\{0_{2}\right\}$ that we get if we adjoint a new element $0_{2}$ to the set $S_{1}$. Define an operation $*$ on $S_{2}$ by setting $a * b=a \cdot b$ if $a, b$ are in $s_{1}$, and $0_{2} * a=a * 0_{2}=0_{2}$ for all a in $S_{2}$. In particular $0_{2} * 0_{1}=0_{1} * 0_{2}=$ $=0_{2}$. We observe then that:

- $\left(\mathrm{S}_{2}, *\right)$ is a semigroup and $0_{2}$ is its zero (absorbent) element (self evident)

- $\left(S_{2}\right.$, *) has no zero divisors. Indeed if $a, b \in S_{2}$ with $a \neq 0_{2} \quad b \neq 0_{2}$ then $a, b \in S_{1}$ and by definition $a * b=a b \in S_{1}$ and hence $a b \neq 0_{2}$.

- $\left(\mathrm{S}_{2}, *\right)$ is non cancellative. Indeed we can take $a, b$ in $\mathrm{S}_{1}$ with $\mathrm{a} \neq \mathrm{b}$. Then $0_{1}{ }^{*} \mathrm{a}=0_{1} \mathrm{a}$ $=0_{1}=0_{1} b=0_{1} * b$. Thus $0_{1} * a=0_{1} * b$ but $a \neq b$.

- Finally we define a total order $\&$ on $\mathrm{S}_{2}$ by setting $a f{ }_{2}$ for all a in $\mathrm{S}_{1}$, and for $\mathrm{a}, \mathrm{b}$, in $\mathrm{S}_{1}, a \& b \Longleftrightarrow \mathrm{b}>\mathrm{b}$. It is obvious that this is well defined, and that $\left(\mathrm{S}_{2}, *, f\right)$ becomes a totally ordered semigroup.

3. A PROPOSITION

Notation:In what follows, we will denote by $S_{1}$ an arbitrary given totally ordered semigroup, and by $\mathrm{S}_{2}$ the corresponding totally ordered non cancellative semigroup without zero divisors, obtained from $s_{1}$, by adjoining a new absorbent element $0_{2}$, as it was done in section 2 .

PROPOSITION: Let $I$ be a two sided ideal of a (not necessarilly commutative) integral domain $R$. If $R / I$ can be hypervaluated by $S_{1}$, then $R$ can be hypervaluated by $S_{2}$.

PROOF: Let $|\ldots|: R / I \rightarrow S_{1}=\left\{0_{1}, a, b, \ldots\right\}$ be a valuation from $R / I$ onto $S_{1}$. We define the function $\|\quad\|: R \rightarrow S_{2}$ by setting: For $a$ in $R,\|a\|=0_{2}$ if $a=0$ and $\|a\|=|a+I|$ if $a \neq 0$. This implies that if $a$ is in $I$, with $a \neq 0$, then $\|a\|=0{ }_{1}$.

We see that $\|.$.$\| thus defined,satisfies the four properties of hypervaluation:$ Indeed properties (1) and (2) of definition 2 are obviously satisfied. That (3) holds for $a l 1 \mathrm{a}, \mathrm{b}$ in $\mathrm{R}$ is immediate if at least one of them equals to zero. So we may assume $a \neq 0, b \neq 0$, and thus $a b \neq 0$ since $R$ is an integral domain. Then $\|a b\|=|a b+I|=|(a+I)(b+I)|=$ $=|a+I||b+I|=\|a\| \div\|b\|$.

Finally (4) is also satisfied. For if $a, b, \in R$, if at least one of them equals to zero the proof is immediate. Suppose now $a, b \neq 0$. Then we could have $a+b=0$ or $a+b \neq 0$. If $a+b=0$ then $\|a+b\|=0_{2}-\boldsymbol{a}\|\cdot \vec{a}\|$, $\|\mathrm{b}\| \underline{\mathbf{s}} \operatorname{lax}\{\|, a\|,\|b\|\}$. If $a+b \neq 0$ then $\|a\|=|a+I|$, $|b \|=| b+I \mid$ and $\|a+b\|=|a+b+I|=|(a+I)+(b+I)| \leq \operatorname{Max}\{|a+I|,|b-r I|\}=\operatorname{Max}\{\|a\|,\|b\|\}$ This completes the proof.

4. COFFIS THEOREM FOR HYPERVALUABILITY OF A RING

DEFINITION 3. Ler $R$ be a ring. For any element a in $R$ we call the set of left annihilators of a to be the set $\{x \in R \mid x \cdot a=0\}$ and we denote this set by $A_{1}(a)$. In an analogous way we define the set of right annihilators of a denoted by $A_{r}(a)$.

THEOREM 1: (Coffi - Nikestia): Let $\mathrm{R}$ be a ring with a untt element 1 . $\mathrm{R}$ can be hypervaluated by a totally ordered semigroup $S$ if and only if it satisfies the following conditional:

1. For $a 11$ a $\varepsilon R, A_{1}(a)=A_{r}(a)$ and we denote this set by $A(a)$.

2. For $a 11 a, b \in R, A(a \cdot b)=A(b . a)$

3. The class $C=\{A(a), A \in R\}$ is totally ordered by inclusion. 
In particular, $R$ posesses an hypervaluation $|\ldots|$ such that $|a| \rightarrow A(a)$ is a one-to-one correspondence between $\mathrm{S}$ and $\mathrm{C}$.

We remark that Coffi in his construction supposes the semigroup to be commutative. The ring $R$ is not supposed to be necessarilly commutative,but with an identity element 1 . The details can be found in Coffi [l]. The idea is the following:For each a in $R$, its "value" $|\mathrm{a}|$ is $\mathrm{A}(\mathrm{a})$. So $|\ldots|: \mathrm{R} \rightarrow \mathrm{C}=\mathrm{S}$. Moreover $\mathrm{S}$ is totally ordered by the total order defined as follows: For $a, b$ in $R|a| \leq|b|$ if $A(a) \supseteq A(b)$.

5. OUR MAIN THEOREM

THEOREM: There exists a totally ordered non cancellative semigroup $S$ without zero divisors, and a ring $\mathrm{R}$ that can be hypervaluated by this semigroup.

PROOF: We choose an integral domain $R$ (not necessarily commutative) such that $R / I$ (for some two-sided ideal $I$ of $R$ ) be a ring satisfying the conditions of Coffi's theorem. Then by Coffi's theorem $\mathrm{R} / \mathrm{I}$ can be hypervaluated by a totally ordered semigroup $\mathrm{S}_{1}$. From $S_{1}$ we obtain a totally ordered, non cancellative semigroup $\mathrm{S}_{2}$ without zero divisors, as we did in section 2 .

By our Proposition 1 , we can hypervaluate $R$ by $S_{2}$ that has the desired properties. This concludes the proof of our theorem.

\section{A CONCRETE EXAMPLE}

We provide in this paragrapha concrete example of a Ring hypervaluated by a totally ordered, non cancellative semigroup $\mathrm{S}_{2}$ without zero divisors.

Jet $Z$ be the ring of integers and (16) the ideal in $Z$ generated by 16 . It suffices to show that the ring $\mathrm{Z} /(16)$ satisfies the conditions of Coffi's theorem and thus can be hypervaluated by a totally ordered semigroup $S_{1}$. Because then, by our Proposition of section $3, \mathrm{Z}$ can be hypervaluated by a semigroup $\mathrm{s}_{2}$, having the desired properties. Indeed since $Z /(16)$ is commutative, conditions 1 and 2 are obviously satisfied. Now if $a, b, x \in Z$ and $\bar{a}, \bar{b}, \bar{x} \in Z /(16)$ their corresponding equivalence classes, $\bar{x}$ is then an annihilator of $\bar{a}$ in $Z /(16)$ if and only if $x . a \in(16)$ i.e.iff 16 divides $x a$. Let (a,b) denote the least common multiple of two elements $a, b$ in $\mathrm{Z}$.

Thus: $\quad$ If $(a, 16)=1$ then $A(\bar{a})=\{\overline{16}\}=\{\overline{0}\}$

If $(a, 16)=2$ then $A(\bar{a})=\{\overline{8}, \overline{16}\}$

If $(a, 16)=4$ then $A(\bar{a})=\{\overline{4}, \overline{8}, \overline{12}, \overline{16}\}$

If $(a, 16)=8$ then $A(\bar{a})=\{\overline{2}, \overline{4}, \overline{6}, \overline{8}, \overline{10}, \overline{12}, \overline{14}, \overline{16}\}$

If in general $(a, 16)=(b, 16)$ then $A(\bar{a})=A(\bar{b})$, if $(a, 16)>(b, 16)$ then $A(\bar{a}) \supset A(\bar{b})$.

Condition 3 of Coffi's theorem is also therefore satisfied.

\section{REFERENCES}

1. COFFI-NIKESTIA J.B. "Valuation des anneau avec diviseurs de zero au moyen des demigroupes totalement ordonnes. Proprietes des anneaux valuables". Compte-Rendus, Acad.Sci.Paris 254 III, 1962. 


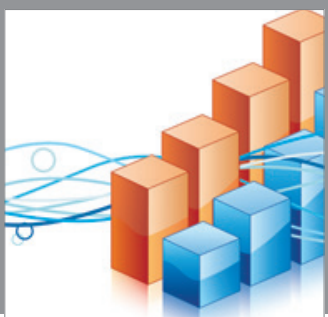

Advances in

Operations Research

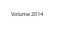

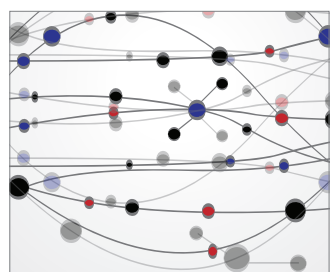

\section{The Scientific} World Journal
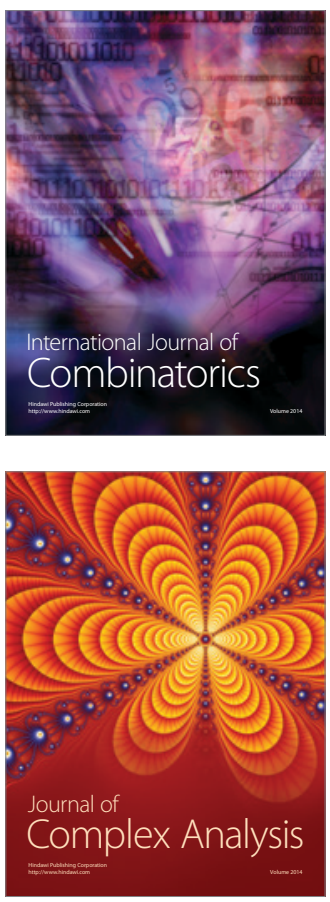

International Journal of

Mathematics and

Mathematical

Sciences
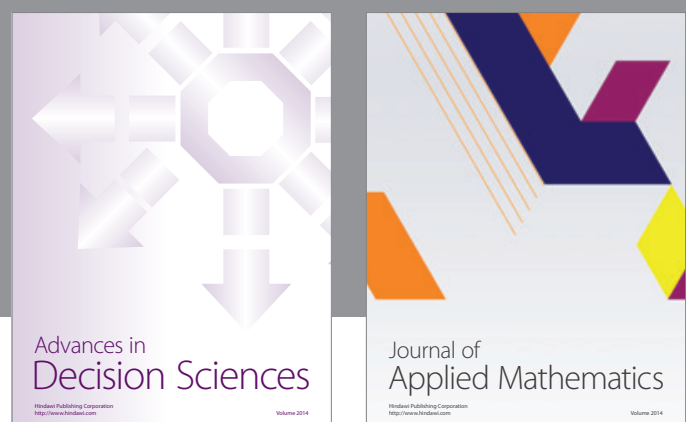

Journal of

Applied Mathematics
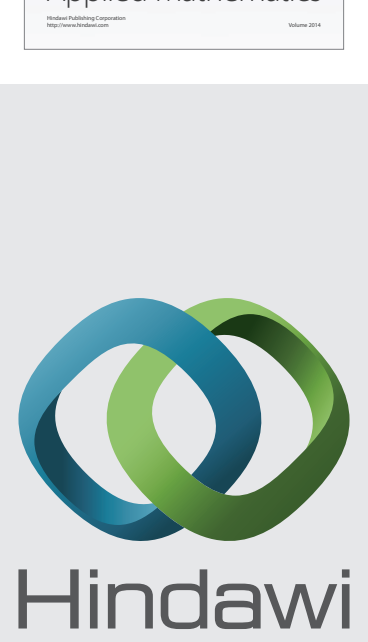

Submit your manuscripts at http://www.hindawi.com
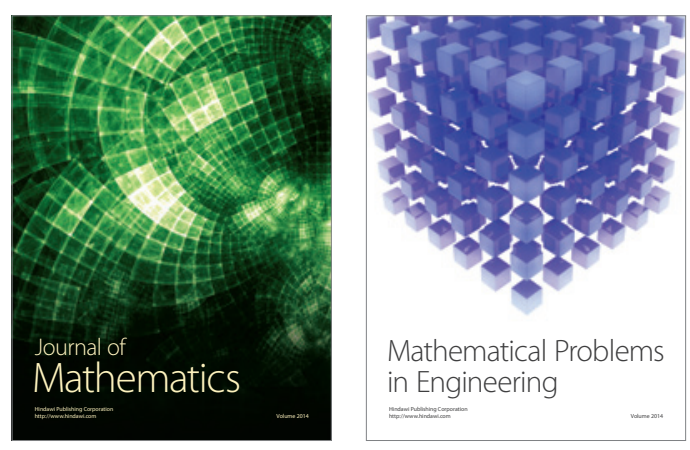

Mathematical Problems in Engineering
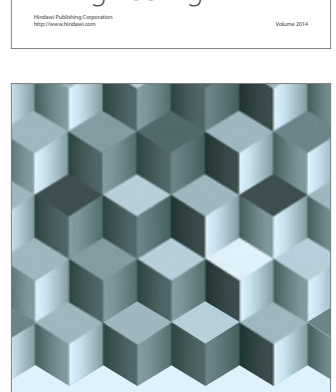

Journal of

Function Spaces
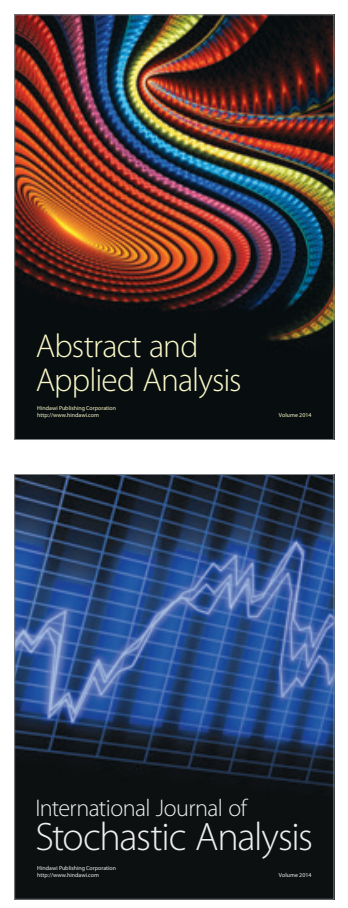

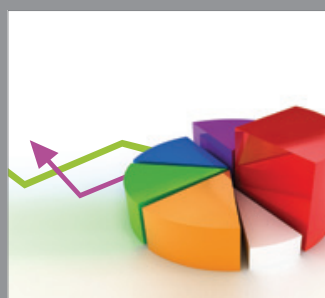

ournal of

Probability and Statistics

Promensencen
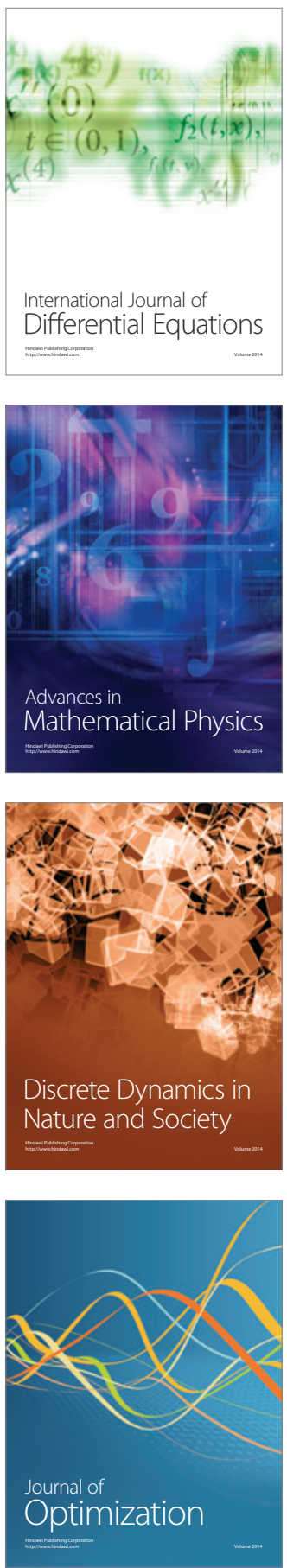